\title{
Transient Analysis and Modelling of Sixphase Asynchronous Machine
}

\author{
Akpama Eko James ${ }^{1}$, Linus Anih ${ }^{2}$, Ogbonnaya Okoro ${ }^{3}$ \\ ${ }^{1}$ Dept. of Elect/Elect/ Engineering Cross River University of Technology, Calabar/Nigeria \\ ${ }^{2}$ Dept. of Electrical Engineering Nniversity of Nigeria, Nsukka, Enugu/Nigeria \\ ${ }^{3}$ Dept. of Elect/Elect/ Engineering, Micheal Okpara University Agriculture, Umudike/Nigeria
}

Email address:

ekoakpama2004@yaahoo.com (E. J. Akpama).luanih@yahoo.com (L. U. Anih). oiokoro@yahoo.co.uk (O. I. Okoro)

\section{To cite this article:}

Akpama Eko James, Linus Anih, Ogbonnaya Okoro. Transient Analysis and Modelling of Sixphase Asynchronous Machine. American Journal of Electrical Power and Energy Systems. Vol. 4, No. 6, 2015, pp. 77-83. doi: 10.11648/j.epes.20150406.11

\begin{abstract}
Multiphase Induction machine offers numerous advantages compared to the conventional three phase induction machine. Among the different types, the six-phase induction machine is most common, due to the flexibility in converting a three phase to six phase. By splitting the phase belt of a three phase induction machine, a six-phase induction machine is realized. The areas of application of multiphase $(>3)$ machines are enormous, mostly where reliability is paramount. This paper presents the analysis, modeling and simulation of a $5 \mathrm{Hp}, 50 \mathrm{HZ}, 4$ pole, 24 slots asymmetrical six-phase induction machine for submarine application. With the help of MATLAB software, the models developed are simulated and results in the form of computer traces of the dynamic performance of the machine at start-up are presented and discussed. This investigation shows that the replacement of three phase with a six phase machine is both technically and economically advantageous.
\end{abstract}

Keywords: Modeling, Six-Phase, Submarines, Computer Simulation, MATLAB, Transients Studies

\section{Introduction}

Three phase induction machines are asynchronous speed machines. They are operated as motors and generators, comparatively less expensive to equivalent synchronous or $\mathrm{dc}$ machines. It is considered as the workhorse of the industry. Induction machines has almost replaced the DC machine in the industry due to the simplicity of design, ruggedness, low-cost, low maintenance cost and direct connection to $\mathrm{AC}$ power source compared to the DC motors[1, 2, 3]. In fact $85 \%$ of the total power consumption in the industrial sector is from induction motors. Due to the above, new strategies, new methods of analysis and design are being sought that will improve the performance of this machines and increase efficiency. Investigation has shown that multiphase machines are possible and advantageous [4]. Among the groups of multiphase machines, the six-phase has received more attention due the simplicity in converting a three phase machine to six-phase machine [5]. This is achieved by splitting the phase belt of three phase machine, that is, two sets of three phase stator winding of the original three phase, with set I spanning $30^{\circ}$ electrical from set II, having a common magnetic structure[6-7].
The use of a common magnetic structure shared by two sets of stator winding dated since 1930 [4, 8], where in an attempts to increase the power capability of a large synchronous generator, the stator winding has to be doubled. From that time, research activities of the dual stator induction machine (DSIM) increased. These type of machines are normally constructed by "splitting" the stator winding into two identical windings [9-12]. Phase orders of multiples of 3, i.e. 6,9, etc, are very possible. Presently, there is evidence of current research on higher phase $\mathrm{AC}$ machines of phase order 5, 6, 9 even 15 [13-15]. Multiphase induction machine finds its applicability in the area of high degree of reliability demand as in more electric aircrafts, electric ship propulsion, electric vehicles, (EH) and hybrid electric vehicles (HEV).

Particularly for ship propulsion, there is an ongoing research in the application of six phase induction motor by this author, where a six phase motor is to replace two three phase motors.

Analysis of multiphase induction machine is carried out in $[16,17]$. Lipo in [8] presented a six-phase induction machine model in $\mathrm{d}-\mathrm{q}$ transformation, the analysis here uses a split-phase configuration where the $60^{\circ}$ phase belt was split into two portions each spanning $30^{\circ}$. The simulated results for 
three phase and six-phase were compared. B. Kundrotas et al [12] modelled a six phase induction motor in the dynamic mode. In [18], a six-phase induction motor was used to reduce the noise level in an electric traction system. Improved reliability is guaranteed in submarine with the application of two sets of star connected stator winding spatially shifted by $30^{0}$ electrical with isolated neutral point as reported in [19]. A transition design based on the Markov chain was used to analyse the availability index of multiphase induction machine, $[20,21]$. The submarine is rapidly emerging in the last couple of years as the main potential application area for multi-phase motor drives. The idea of multiphase suggests a replacement of two electric machines with a single machine of DSIM. A lot of research is ongoing in multiphase induction machines. The concept of multiphase is still in the infant stage, though much research is ongoing. It is the aim of this paper to investigate the transient performance of a six-phase split wound induction machine for submarines.

\section{Modelling of Six-Phase Induction Machine}

Multiphase electric machine (MPEM) which is more advantageous and has improved performance compared to the three phase counterpart come as both synchronous and asynchronous machines. Figure 1 , shows the $\mathrm{V}_{\text {abcxyz }}$ voltages of a six-phase induction machine with their phase displacement. In order to model the six-phase induction machine, the dq transformation is applied, and the rotor reference frame is adopted [16].

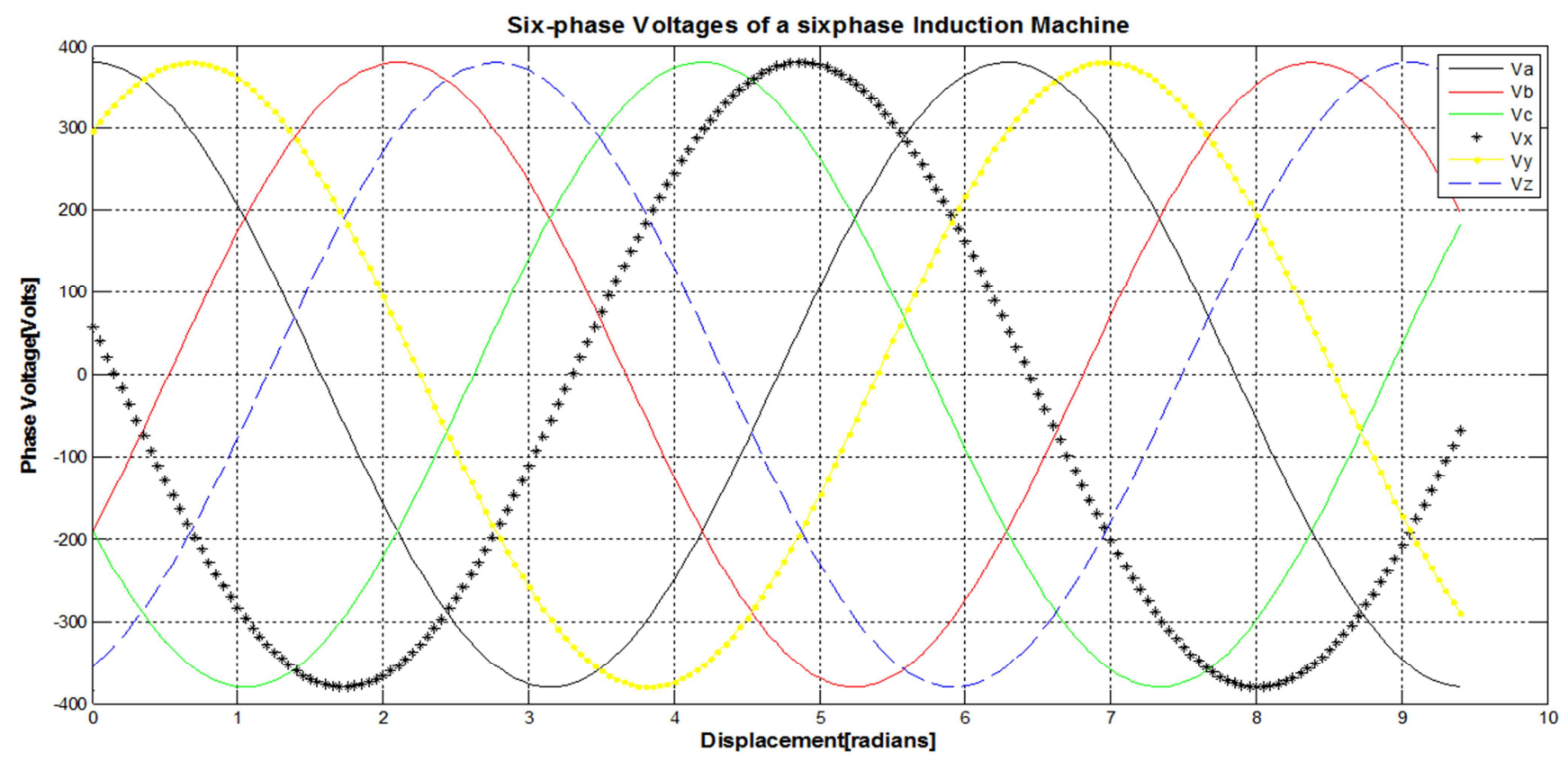

Fig. 1. Six-phase source voltages of six-phase induction machine.

\subsection{Electrical Model}

The voltage equation of a split phase induction machine is written as, where $\alpha=30^{\circ}$ elect

$$
\begin{aligned}
\mathrm{V}_{\mathrm{as}} & =\mathrm{V} \cos \omega_{\mathrm{e}} \mathrm{t} \\
\mathrm{V}_{\mathrm{bs}} & =\mathrm{V} \cos \left(\omega_{\mathrm{e}} \mathrm{t}-2 \pi / 3\right) \\
\mathrm{V}_{\mathrm{cs}} & =\mathrm{V} \cos \left(\omega_{\mathrm{e}} \mathrm{t}+2 \pi / 3\right) \\
\mathrm{V}_{\mathrm{xs}} & =\mathrm{V} \cos \left(\omega_{\mathrm{e}} \mathrm{t}-\alpha\right) \\
\mathrm{V}_{\mathrm{ys}} & =\mathrm{V} \cos \left(\omega_{\mathrm{e}} \mathrm{t}-2 \pi / 3-\alpha\right) \\
\mathrm{V}_{\mathrm{zs}} & =\mathrm{V} \cos \left(\omega_{\mathrm{e}} \mathrm{t}+2 \pi / 3-\alpha\right)
\end{aligned}
$$

Using the appropriate transformation, the phase voltage of set I, abc, is transformed to its equivalent d-q axis as below;

$$
\mathrm{V}_{\mathrm{qs}}=2 / 3\left(\mathrm{~V}_{\mathrm{as}}-\mathrm{V}_{\mathrm{bs}} / 2-\mathrm{V}_{\mathrm{cs}} / 2\right)
$$

$$
\mathrm{V}_{\mathrm{ds}}=2 / 3\left(\sqrt{3} / 2\left[-\mathrm{V}_{\mathrm{bs}}-\mathrm{V}_{\mathrm{cs}}\right]\right)
$$

For a balance system, since

$$
\mathrm{V}_{\mathrm{as}}=-\mathrm{V}_{\mathrm{bs}}-\mathrm{V}_{\mathrm{cs}}
$$

Then equation (7) becomes

$$
\mathrm{V}_{\mathrm{qs}}=\mathrm{V}_{\mathrm{as}}=\mathrm{V} \cos \omega_{\mathrm{e}} \mathrm{t}
$$

Simplifying equation (8)

$$
\begin{gathered}
\mathrm{V}_{\mathrm{ds}}=1 / \sqrt{3}\left(\mathrm{~V}_{\mathrm{cs}}-\mathrm{V}_{\mathrm{bs}}\right) \\
\mathrm{V}_{\mathrm{ds}}=1 / \sqrt{3}\left(\mathrm{~V} \cos \left(\omega_{\mathrm{e}} \mathrm{t}+2 \pi / 3\right)-\mathrm{V} \cos \left(\omega_{\mathrm{e}} \mathrm{t}-2 \pi / 3\right)\right.
\end{gathered}
$$

Applying Euler's identity to equation (9), the result becomes

$$
\mathrm{V}_{\mathrm{ds}}=-\mathrm{V} \sin \omega_{\mathrm{e}} \mathrm{t}
$$


The same analysis is carried out on the second set II. The dq voltage equations of a six-phase induction machine are readily written as in [24]:

$$
\begin{aligned}
& \mathrm{V}_{\mathrm{q} 1}=\mathrm{r}_{1} \mathrm{i}_{\mathrm{q} 1}+\omega_{\mathrm{k}} \lambda_{\mathrm{d} 1}+\mathrm{p} \lambda_{\mathrm{q} 1} \\
& \mathrm{~V}_{\mathrm{d} 1}=\mathrm{r}_{1} \mathrm{i}_{\mathrm{d} 1}-\omega_{\mathrm{k}} \lambda_{\mathrm{q} 1}+\mathrm{p} \lambda_{\mathrm{d} 1} \\
& \mathrm{~V}_{\mathrm{q} 2}=\mathrm{r}_{2} \mathrm{i}_{\mathrm{q} 2}+\omega_{\mathrm{k}} \lambda_{\mathrm{d} 2}+\mathrm{p} \lambda_{\mathrm{q} 2} \\
& \mathrm{~V}_{\mathrm{d} 2}=\mathrm{r}_{2} \mathrm{i}_{\mathrm{d} 2}-\omega_{\mathrm{k}} \lambda_{\mathrm{q} 2}+\mathrm{p} \lambda_{\mathrm{d} 2} \\
& \mathrm{~V}_{\mathrm{qr}}=0=\mathrm{r}_{\mathrm{r}} \mathrm{i}_{\mathrm{qr}}+\left(\omega_{\mathrm{k}}-\omega_{\mathrm{r}}\right) \lambda_{\mathrm{dr}}+\mathrm{p} \lambda_{\mathrm{qr}} \\
& \mathrm{V}_{\mathrm{dr}}=0=\mathrm{r}_{\mathrm{r}} \mathrm{i}_{\mathrm{dr}}-\left(\omega_{\mathrm{k}}-\omega_{\mathrm{r}}\right) \lambda_{\mathrm{qr}}+\mathrm{p} \lambda_{\mathrm{dr}}
\end{aligned}
$$

The flux linkage equations are given below

$$
\begin{aligned}
& \lambda_{\mathrm{q} 1}=\mathrm{L}_{11} \mathrm{i}_{\mathrm{q} 1}+\mathrm{L}_{\mathrm{lm}}\left(\mathrm{i}_{\mathrm{q} 1}+\mathrm{i}_{\mathrm{q} 2}\right)+\mathrm{L}_{\mathrm{dq}} \mathrm{i}_{\mathrm{d} 2}+\mathrm{L}_{\mathrm{mq}}\left(\mathrm{i}_{\mathrm{q} 1}+\mathrm{i}_{\mathrm{q} 2}+\mathrm{i}_{\mathrm{qr}}\right) \\
& \lambda_{\mathrm{d} 1}=\mathrm{L}_{11} \mathrm{i}_{\mathrm{d} 1}+\mathrm{L}_{\mathrm{lm}}\left(\mathrm{i}_{\mathrm{d} 1}+\mathrm{i}_{\mathrm{d} 2}\right)+\mathrm{L}_{\mathrm{dq}} \mathrm{i}_{\mathrm{i} 2}+\mathrm{L}_{\mathrm{md}}\left(\mathrm{i}_{\mathrm{d} 1}+\mathrm{i}_{\mathrm{d} 2}+\mathrm{i}_{\mathrm{dr}}\right) \\
& \lambda_{\mathrm{q} 2}=\mathrm{L}_{12} \mathrm{i}_{\mathrm{q} 2}+\mathrm{L}_{\mathrm{lm}}\left(\mathrm{i}_{\mathrm{q} 1}+\mathrm{i}_{\mathrm{q} 2}\right)+\mathrm{L}_{\mathrm{dq}} \mathrm{i}_{\mathrm{d} 1}+\mathrm{L}_{\mathrm{mq}}\left(\mathrm{i}_{\mathrm{q} 1}+\mathrm{i}_{\mathrm{d} 2}+\mathrm{i}_{\mathrm{qr}}\right) \\
& \lambda_{\mathrm{d} 2}=\mathrm{L}_{\mathrm{l} 2} \mathrm{i}_{\mathrm{d} 2}+\mathrm{L}_{\mathrm{lm}}\left(\mathrm{i}_{\mathrm{d} 1}+\mathrm{i}_{\mathrm{d} 2}\right)+\mathrm{L}_{\mathrm{dq}} \mathrm{i}_{\mathrm{q} 1}+\mathrm{L}_{\mathrm{md}}\left(\mathrm{i}_{\mathrm{d} 1}+\mathrm{i}_{\mathrm{d} 2}+\mathrm{i}_{\mathrm{dr}}\right) \\
& \lambda_{\mathrm{qr}}=\mathrm{L}_{\mathrm{lr}} \mathrm{i}_{\mathrm{qr}}+\mathrm{L}_{\mathrm{mq}}\left(\mathrm{i}_{\mathrm{q} 1}+\mathrm{i}_{\mathrm{q} 2}+\mathrm{i}_{\mathrm{qr}}\right) \\
& \lambda_{\mathrm{dr}}=\mathrm{L}_{\mathrm{lr}} \mathrm{i}_{\mathrm{dr}}+\mathrm{L}_{\mathrm{md}}\left(\mathrm{i}_{\mathrm{d} 1}+\mathrm{i}_{\mathrm{d} 2}+\mathrm{i}_{\mathrm{dr}}\right)
\end{aligned}
$$

Equations 14-25, suggested the equivalent circuit of Fig. 2

Let

$$
\begin{aligned}
& \mathrm{Ldq}=0 \\
& \mathrm{~L}_{\mathrm{m}}=\mathrm{L}_{\mathrm{mq}}=\mathrm{L}_{\mathrm{md}} \\
& \mathrm{L}_{1}=\mathrm{L}_{11}+\mathrm{L}_{\mathrm{lm}}+\mathrm{L}_{\mathrm{m}}
\end{aligned}
$$

$$
\begin{aligned}
& \mathrm{L}_{2}=\mathrm{L}_{12}+\mathrm{L}_{\mathrm{lm}}+\mathrm{L}_{\mathrm{m}} \\
& \mathrm{L}_{3}=\mathrm{L}_{\mathrm{lm}}+\mathrm{L}_{\mathrm{m}} \\
& \mathrm{L}_{\mathrm{r}}=\mathrm{L}_{\mathrm{lr}}+\mathrm{L}_{\mathrm{m}}
\end{aligned}
$$

Using state variable method, equation (32) is put in state variable form [17];

$$
\begin{aligned}
& {\left[\begin{array}{c}
V_{q 1} \\
V_{d 1} \\
V_{q 2} \\
V_{d 2} \\
V_{q r} \\
V_{d r}
\end{array}\right]=\left[\begin{array}{c}
r_{1} \omega_{k} L_{1} 0 \omega_{k} L_{2} 0 \omega_{k} L_{m} \\
-\omega_{k} L_{1} r_{1}-\omega_{k} L_{2}-0_{2}-w_{k} L_{m} \\
0 \omega_{k} L_{3} r_{2} \omega_{k} L_{2} 0 \omega_{k} L_{m} \\
-\omega_{k} L_{3} 0-\omega_{k} L_{2} r_{2}-\omega_{k} L_{m} 0 \\
0 \alpha L_{m} 0 \alpha L_{m} r_{r} \alpha L_{r} \\
-\alpha L_{m} 0-\alpha L_{m} 0-\alpha L_{r} r_{r}
\end{array}\right]\left[\begin{array}{c}
i_{q 1} \\
i_{d 1} \\
i_{q 2} \\
i_{d 2} \\
i_{q r} \\
i_{d r}
\end{array}\right]+\left[\begin{array}{c}
P i_{q 1} \\
P i_{d 1} \\
P i_{q 2} \\
P i_{d 2} \\
P i_{q r} \\
P i_{d r}
\end{array}\right]} \\
& {\left[\begin{array}{cccccc}
L_{1} & 0 & L_{2} & 0 & L_{m} & 0 \\
0 & L_{1} & 0 & L_{2} & 0 & L_{m} \\
L_{3} & 0 & L_{2} & 0 & L_{m} & 0 \\
0 & L_{3} & 0 & L_{2} & 0 & L_{m} \\
L_{m} & 0 & L_{m} & 0 & L_{r} & 0 \\
0 & L_{m} & 0 & L_{m} & 0 & L_{r}
\end{array}\right]\left[\begin{array}{l}
P i_{q 1} \\
P i_{d 1} \\
P i_{q 2} \\
P i_{d 2} \\
P i_{q r} \\
P i_{d r}
\end{array}\right]} \\
& \operatorname{idot}=[L][V]-[L]^{-1}[G][1]
\end{aligned}
$$

where,

$$
\begin{gathered}
{[V]=\left[\begin{array}{llll}
V_{q 1} V_{d 1} V_{q 2} & V_{d 2} & V_{q r} & V_{d r}
\end{array}\right]} \\
{[I]=\left[\begin{array}{lll}
i_{q 1} i_{d 1} i_{q 2} i_{d 2} & i_{q r} i_{d r}
\end{array}\right]}
\end{gathered}
$$$$
[G]=\left[\begin{array}{cccccc}
r_{1} & \omega_{k} L_{1} & 0 & \omega_{k} L_{2} & 0 & \omega_{k} L_{m} \\
-\omega_{k} L_{1} & r_{1} & \omega_{k} L_{2} & 0 & -\omega_{k} L_{m} & 0 \\
0 & \omega_{k} L_{3} & r_{2} & \omega_{k} L_{2} & 0 & \omega_{k} L_{m} \\
-\omega_{k} L_{3} & 0 & -\omega_{k} L_{2} & r_{2} & -\omega_{k} L_{m} & 0 \\
0 & \alpha L_{m} & 0 & \alpha L_{m} & r_{r} & \alpha L_{m} \\
\alpha L_{m} & 0 & -\alpha L_{m} & 0 & -\alpha L_{m} & r_{r}
\end{array}\right]
$$
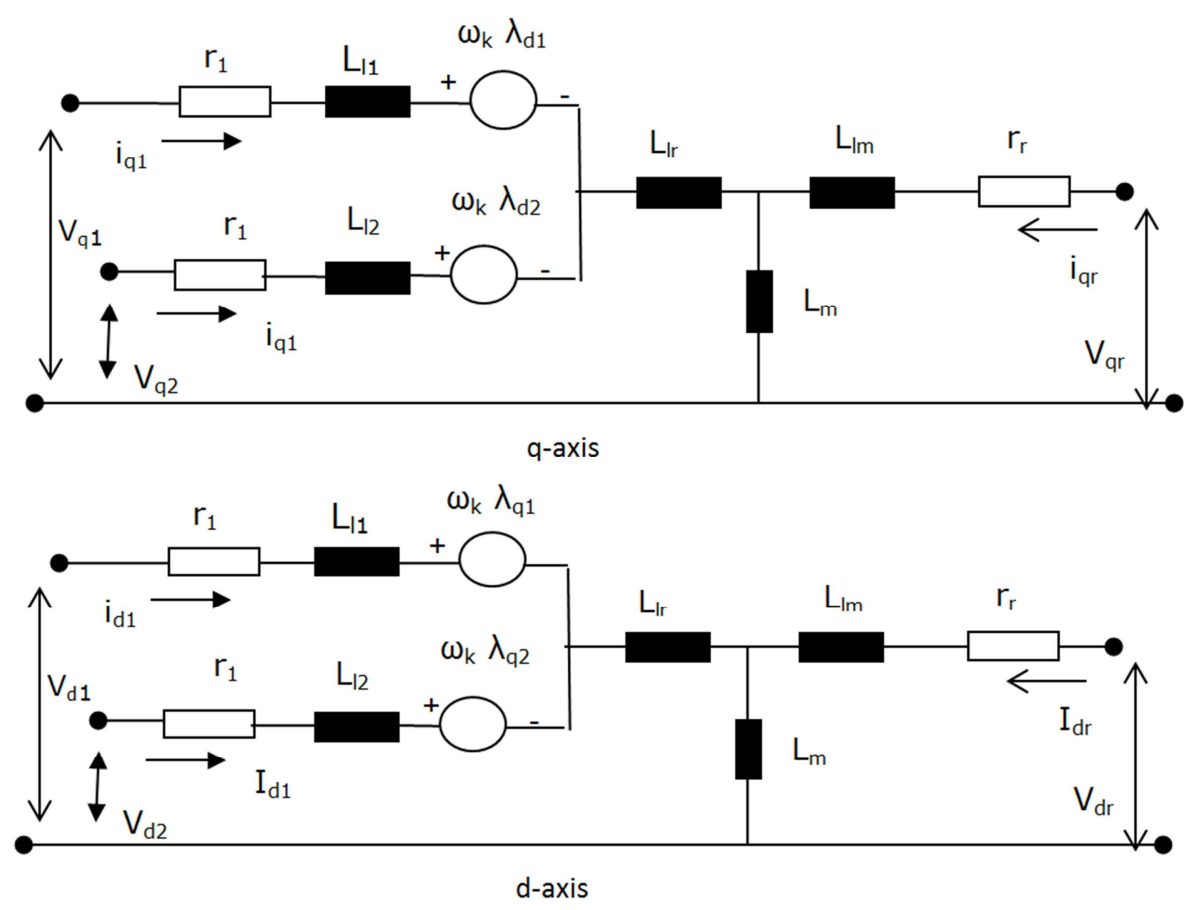

Fig. 2. DQ equivalent circuit of a six-phase Induction Machine. 
Where

$$
\begin{aligned}
& \alpha=\omega_{\mathrm{r}}-\omega_{\mathrm{k}} \\
& {[L]=\left[\begin{array}{llllll}
L_{1} & 0 & L_{2} & 0 & L_{m} & 0 \\
0 & L_{1} & 0 & L_{2} & 0 & L_{m} \\
L_{3} & 0 & L_{2} & 0 & L_{m} & 0 \\
0 & L_{3} & 0 & L_{2} & 0 & L_{m} \\
L_{m} & 0 & L_{m} & 0 & L_{r} & 0 \\
0 & L_{m} & 0 & L_{m} & 0 & L_{r}
\end{array}\right]}
\end{aligned}
$$

\subsection{Mechanical Model}

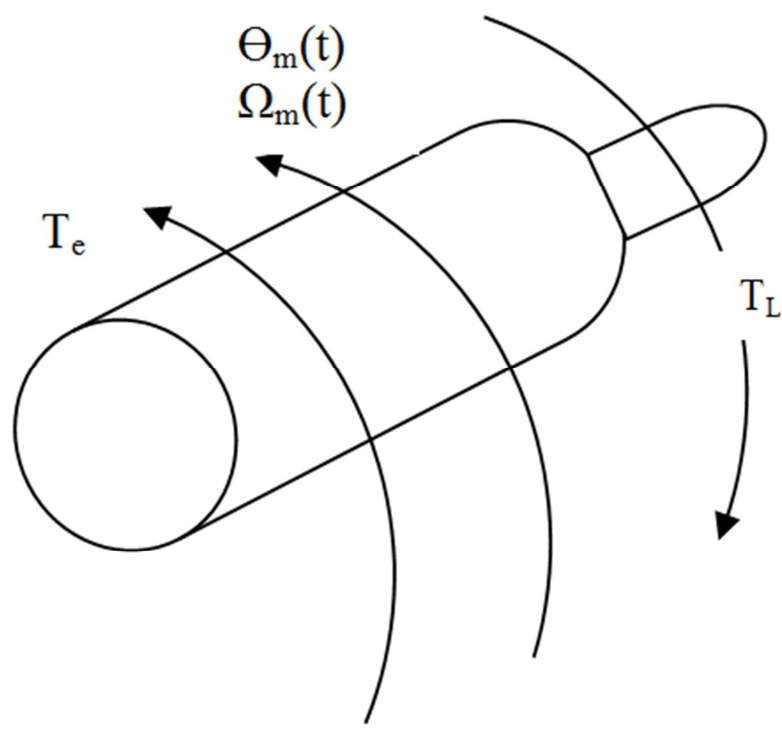

Fig. 3. Induction Motor Mechanical Model.

The mechanical model of the six-phase induction machine is the equation of motion of the machine and driven load as in fig. 3, the figure suggest equation (39),

$$
\mathrm{J}_{\mathrm{m}} \mathrm{p}^{2} \theta_{\mathrm{m}}=\mathrm{T}_{\mathrm{e}}-\mathrm{F} \omega_{\mathrm{r}}-\mathrm{T}_{\mathrm{L}}
$$

The mechanical data of the experimental machine indicates that the combined rotor and load viscous friction ' $F$ ' is appropriately zero, so that, equation (38) becomes.

$$
\mathrm{J}_{\mathrm{m}} \mathrm{p}^{2} \theta_{\mathrm{m}}=\mathrm{T}_{\mathrm{e}}-\mathrm{T}_{\mathrm{L}}
$$

Breaking equation (40) into two first-order differential equation gives

$$
\mathrm{J}_{\mathrm{m}} \mathrm{p}\left(\omega_{\mathrm{m}}\right)=\left(\mathrm{T}_{\mathrm{e}}-\mathrm{T}_{\mathrm{L}}\right)
$$

Because

$$
\mathrm{p} \theta_{\mathrm{m}}=\omega_{\mathrm{m}}
$$

We know that

$$
\omega_{\mathrm{r}}=\omega_{\mathrm{m}} \mathrm{p}
$$

And

$$
\theta_{\mathrm{r}}=\theta_{\mathrm{m}} \mathrm{p}
$$

Where $P=\frac{d}{d t}$, and $\omega_{\mathrm{m}}, \theta_{\mathrm{m}}, \theta_{\mathrm{r},} \omega_{\mathrm{r}}, \mathrm{J}_{\mathrm{m}}$ and $\mathrm{T}_{\mathrm{L}}$ represent angular velocity of the rotor, rotor angular position, electrical rotor angular position, electrical angular velocity, combined rotor and load inertia coefficient, and applied load torque respectively. Matlab m-files are developed to simulate the transient performance of a six-phase, 4 pole, $50 \mathrm{~Hz}$ squirrel cage induction machine.

\section{Experiment}

In order to get data for simulation, a $5.5 \mathrm{Hp} 3 \varnothing, 24$ slot, 4 pole induction motor was reconfigured into a split phase (6-phase) motor with two set of 3-phase displaced $30^{\circ}$ elect from each other. The new motor maintains all specifications of the old, except that, the motor is now a split winding motor (6-phase). The construction motor tested on No-load and On-load. Retardation test was also carried out. The experimental results and the computed results is shown in table 1 .

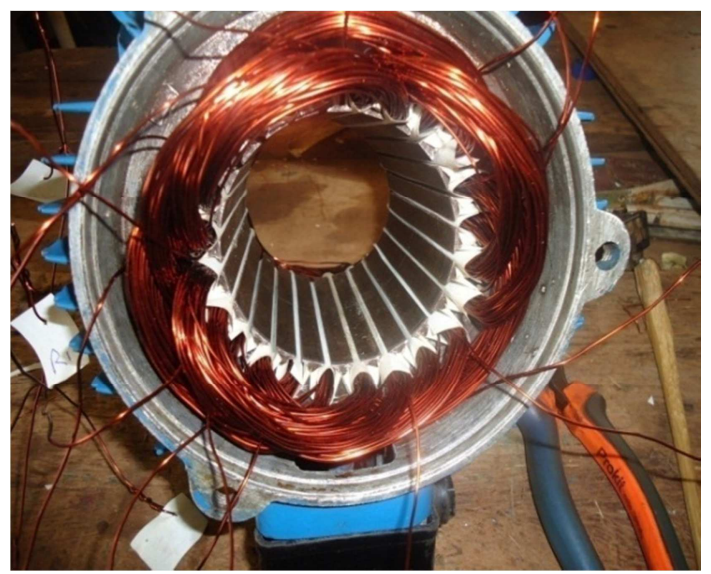

Fig. 4. Stator winding of the sample motor.

Table 1. Simulation Parameters.

\begin{tabular}{ll}
\hline Parameter & Value \\
\hline Power Rating & $5 \mathrm{HP}$ \\
Phase Number & 6 \\
No of Poles & 4 \\
Efficiency & $85 \%$ \\
Power Factor & $0.8 \mathrm{lag}$ \\
Mechanical Speed & $1400 \mathrm{rev} / \mathrm{min}^{-}$ \\
Frequency $(\mathrm{f})$ & $50 \mathrm{~Hz}$ \\
Stator Resistance $\left(\mathrm{R}_{\mathrm{s}}\right)$ & $0.28 \Omega$ \\
Rotor Resistance $\left(\mathrm{R}_{\mathrm{r}}\right)$ & $2.14 \Omega$ \\
Stator winding reactance $\left(\mathrm{X}_{\mathrm{s}}\right)$ & $1.24989 \Omega$ \\
Rotor winding reactance $\left(\mathrm{X}_{\mathrm{r}}\right)$ & $1.24989 \Omega$ \\
Magnetizing reactance $\left(\mathrm{X}_{\mathrm{m}}\right)$ & $35.718 \Omega$ \\
Rotor inertia $\left(\mathrm{J}_{\mathrm{m}}\right)$ & $0.25 \mathrm{Kgm}$ \\
Load inertia $\left(\mathrm{T}_{\mathrm{L}}\right)$ & $0.52 \mathrm{Kgm}$ \\
\hline
\end{tabular}



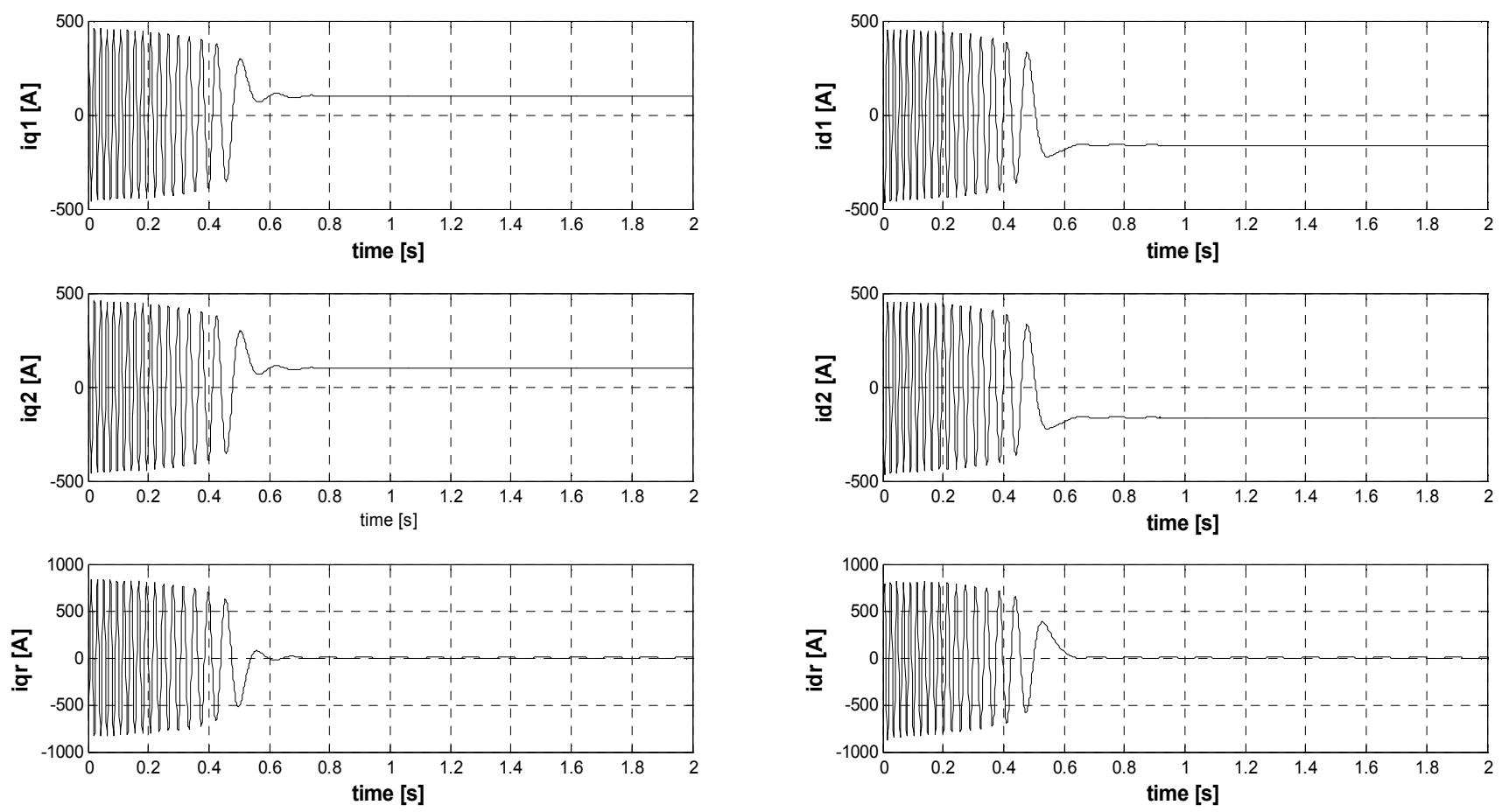

Fig. 5. A graph of dq currents against time.
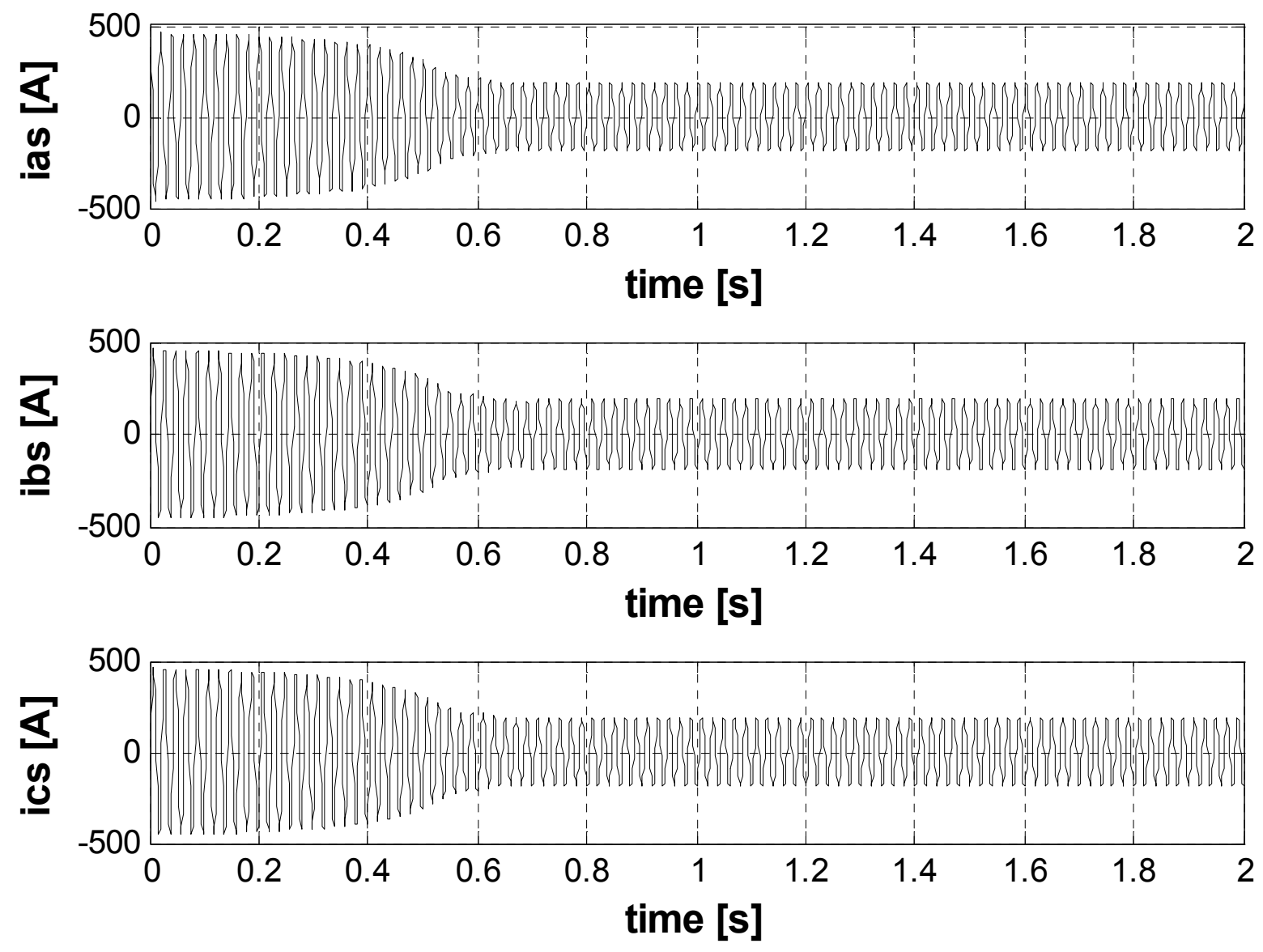

Fig. 6. A graph of xyz phase currents against time. 

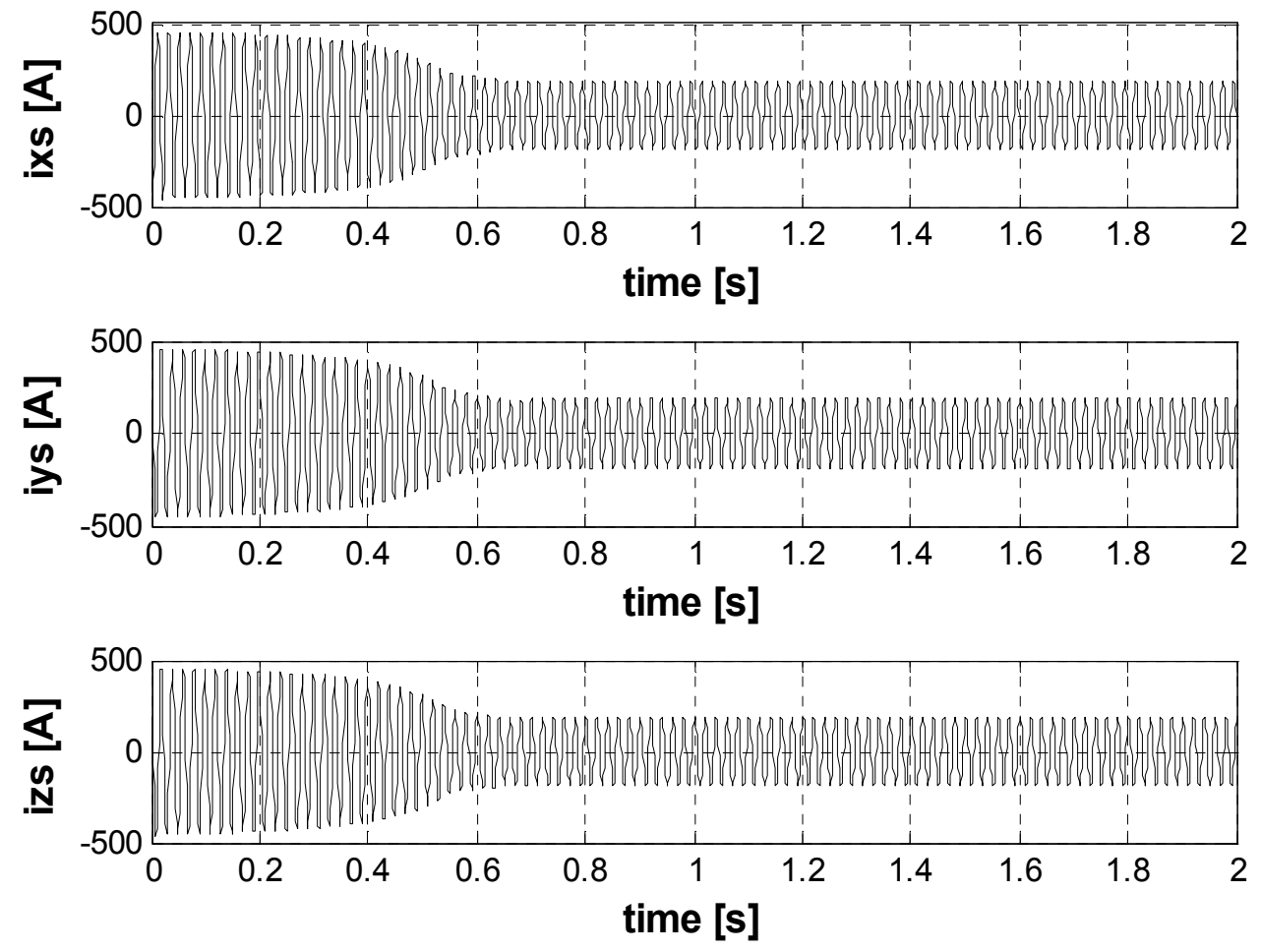

Fig. 7. A graph of xyz phase currents against time.
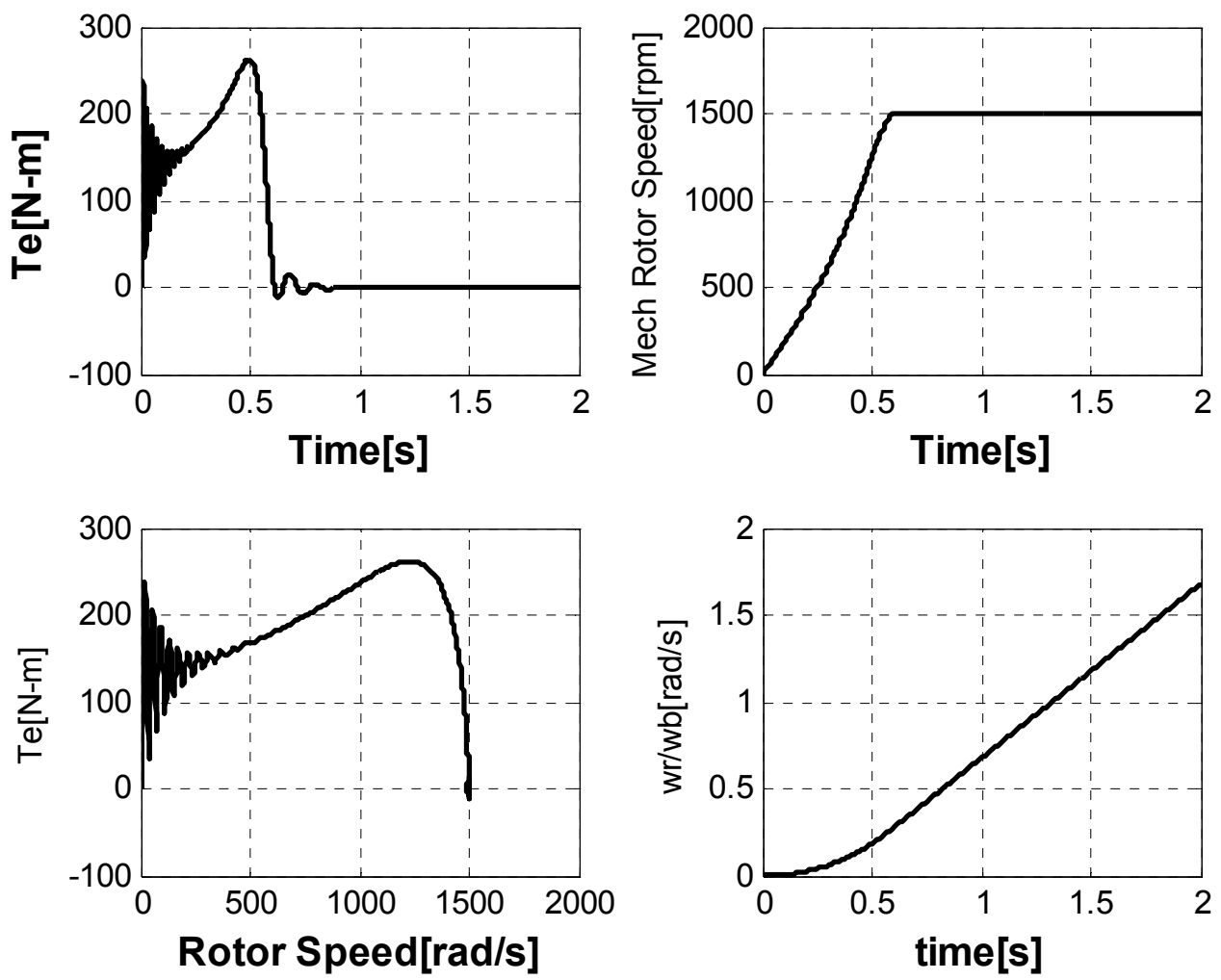

Fig. 8. Mechanical Model plots.

\section{Simulation Results}

The simulation results in the form of computer traces are presented of the performance of a six-phase induction machine is presented in figs 5-8. The simulation for phase currents and qd currents, mechanical rotor speed and Electromagnetic Torque are simulated and presented using the data in table1. 


\section{Discusion of Results}

Electromagnetic Torque in fig. 8 , stabilizes at $0.7 \mathrm{~s}$. The mechanical rotor speed reaches synchronous speed at $0.6 \mathrm{~s}$, this agrees favorably with the theoretical concept. The phase currents also stabilize at $0.6 \mathrm{~s}$. Looking at the simulations, the redesigned motor can work as a three phase machine or/and as six phase. In submarines, the idea here is to use a six phase motor to replace two three phase motors, and money is saved redesigning the machine with higher reliability. The loss of a phase does not stop the motor from running. In submarines mostly Naval War ships, reliability is a criterion in designing a submarines.

\section{Conclusion}

The simulation result shows that, the split six phase is a dual stator induction machine with sets of three phase currents; $I_{a b c}$ and $\mathrm{I}_{\mathrm{xyz}}$. This is expected because DSIM is like paralleling two three phase induction motors. So, instead of using two three phase induction motors for propulsion (submarines), a single DSIM can replace the two and the cost is reduced and reliability increased which is the actual aim of this work.

\section{References}

[1] P.S. Bimbhra, "Generalized theory of electrical machines", Khanna publishers India fourth ed pp. $179-546.1987$

[2] P.C. Krause, "Analysis of electric machinery", MC. Graw Hill book Inc USA 19 S86.

[3] P. C. Krause, F. Nozari, T. L. Skvarenina and D.W. Olive. "The theory of neglecting stator transients", IEEE Trans Power Apparatus and systems vol. 98 pp 141-148, Jan/Feb 1979.

[4] P. L. Alger, et al., "Double windings for turbine alternators," AIEE Transactions, vol. 49, January, 1930, pp.226-244.

[5] Hadiouche D., Razik H., Rezzoug A., "Modelling of a double-star induction motor with an arbitrary shift angle between its three phase winding". $9^{\text {th }}$ international conference on EPE, PEMC 2000 Kosice, Slovak Republic.

[6] K. Gopakumar, et al., "Split-phase induction motor operation from pwm voltage source inverter," IEEE Transactions on Industry Applications, vol. 29, no. 5, Sep./Oct. 1993, pp. 927-932.

[7] Fuchs E. F. and Rosenberg L. T., "Analysis of an Alternator with Two Displaced Stator Windings," IEEE Trans. Power Apparatus and Systems, 1974, vol. 93, pp. 1776-1786.

[8] T.A. Lipo, "A d-q model for six phase induction machines," Proc. Int. Conf. on Electrical Machines ICEM, Athens, Greece, pp. 860-867, 1980.
[9] R.H. Nelson, P.C. Krause, Induction machine analysis for arbitrary displacement between multiple winding sets, IEEE Trans. Power Apparatus Syst. PAS-93 (1974) 841-848.

[10] L.J. Hunt, “A new type of induction motor,” J. Inst Elect. Eng., vol.39, pp. 648-677, 1907.

[11] Singh G. K., "Multi-Phase Induction Machine Drive Research - A Survey," Electric Power Systems Research, 2002, vol. 61, pp. 139-147.

[12] B. Kundrotas, S. Lisauskas, R. Rinkevičienè. Daugiafazio asinchroninio variklio modelis // Elektronika ir elektrotechnika. - Kaunas: Technologija, 2011. - Nr. 5(111). - P. 111-114.

[13] Bugenis S. J., Vanagas J., Gečys S. Optimal phase number of induction motor with the integrated frequency converter // Electronics and Electrical Engineering. - Kaunas: Technologija, 2008. - No. 8(88). - P.67-70.

[14] E.E. Ward, H. H"arer, "Preliminary investigation of an inverter-fed 5-phase induction motor", Proc. IEE 116 (6) (1969) 980-984.

[15] M.A. Abbas, R. Christen, T.M. Jahns, Six-phase voltage source inverter driven induction motor, IEEE Trans. Ind. Appl. IA-20 (5) (1984) 1251-1259.

[16] O.I. Okoro and T.C. Nwodo; "Simulation Tools for electrical machine modeling", Teaching and Research, International conference on power system operation and planning - v1 (ICPSOP), pp. 120 - 124. 2005.

[17] O.I. Okoro and T.C. Nwodo; "Simulation Tools for electrical machine modeling", Teaching and Research, International conference on power system operation and planning - v1 (ICPSOP), pp. 120 - 124. 2005.

[18] E.J. Akpama and O.I.Okoro, "Simulating Asynchronous Machine with Saturation effect", Proceedings of ESPTAEE 2008 National Conference, University of Nigeria Nsukka, pp130-135, June 2008.

[19] A.N. Golubev and S.V. Ignatenko, "Influence of number of stator-winding phases on the noise characteristics of an asynchronous motor," Russian Electrical Engineering, vol. 71, no. 6 , pp. 41-46, 2000.

[20] C. Hodge, S. Williamson and S. Smith, "Direct drive marine propulsion motors," Proc. Int. Conf. on Electrical Machines ICEM, Bruges, Belgium, CD-ROM paper 087, 2002.

[21] M. Molaei, H. Oraee, and M. Fotuhi-Firuzabad, "Markov Model of Drive-Motor Systems for Reliability Calculation," in Proc. IEEE Int. Symp. on Ind. Electron., 2006, pp. 2286-2291.

[22] Jahns T. M., "Improved Reliability in Solid-State AC Drives by Means of Multiple Independent Phase-Drive Units," IEEE Trans. Ind. Application, May/June 1980, vol. IA-16, pp.321-331. 